\title{
Standard Deviation of Wind Direction Estimated from Direct Observation of a Sensitive Wind Vane
}

\author{
J. A. TURNER ${ }^{1}$ \\ Canada Department of Forestry and Rural Development, Vicloria, B. C.
}

18 January 1968

The dispersion of smoke or vapor plumes is strongly dependent on the intensity of atmospheric turbulence. One of the most useful indicators of this dispersive power is provided by the behavior of a sensitive vane.

If an analogue trace is available, the gustiness may be classified according to the method of Singer and Smith (1953), or the standard deviation of suitable short period averages may be calculated (Hay and Pasquill, 1959).

It should be emphasized that the values of the standard deviation of the vane angle are dependent on the response characteristics of the particular vane being used. Consequently, the results from any particular vane can only be compared with those from other vanes having similar response characteristics. If the standard deviation of a vane having perfect response is required, this can be approximated, provided the vane is small enough, if the vane has a damping ratio of about 0.43 (MacCready and Jex, 1964).

In a recent pilot study relating to the dispersion of odor plumes near the ground, gustiness was indicated by direct observation of the fluctuations of a lightweight sensitive vane of balsa wood and a drinking straw, mounted on a needle bearing.

\footnotetext{
1 Permanent affliation: Canadian Meteorological Branch, Department of Transport.
}

The rapid fluctuations of the vane made it impractical to estimate the average direction over any time interval. It was more appropriate to record the position of the vane at each reversal of its swing, and thus obtain the frequency distribution of the vane fluctuations for the given sampling period.

The theoretical relationship between the standard deviation $\sigma_{A}$ of the horizontal wind direction and the average amount of veering or backing on an individual swing of the vane has been shown (Harris and McCormick, 1963) to be

$$
\sigma_{A}=1.2 \theta / N
$$

where $\theta$ is the cumulative veering (or backing) in degrees in the sample period, and $N$ the number of reversals.

The technique of recording the angle at each reversal of swing direction resulted in failure to record an appreciable number of "hesitations" of the vane (where no actual reversal of swing occurred), as well as a few reversals of small amplitude. This produced a bias of undetermined magnitude in estimates of $\sigma_{d}$ calculated by the method of Harris and McCormick.

It was assumed that the distribution of vane oscillations should be approximately Gaussian about a mean value of zero for steady conditions. This was tested by plotting the logarithm of frequency density against the 


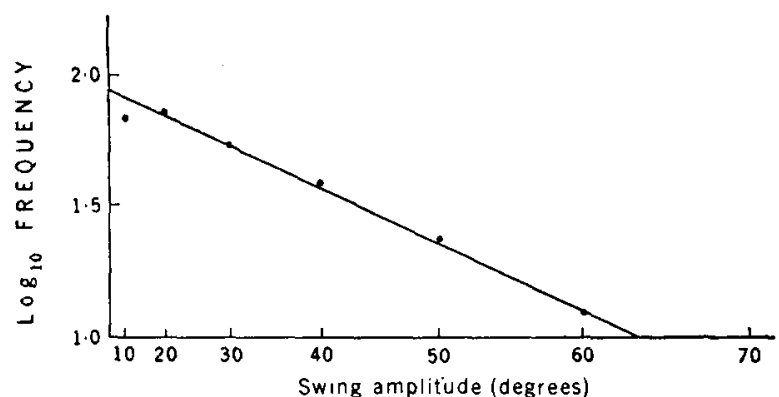

(a) Open site 1920 PDT / Aug. 1, 1967.

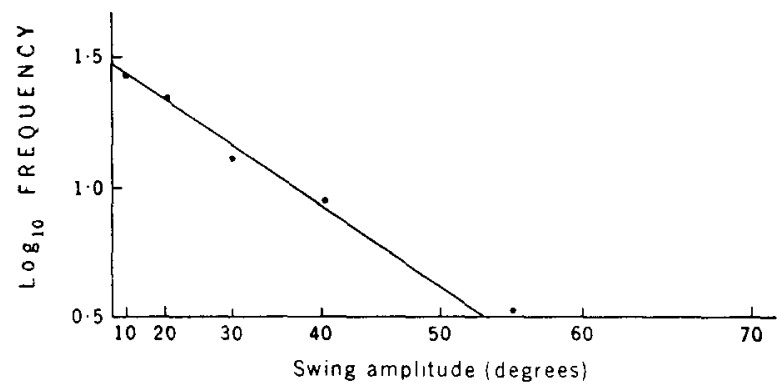

(b) Woods site 1310 PDT / July 25, 1967

FIG. 1. Data illustrating linear relationship between $\log _{10}$ of frequency density of vane oscillations vs swing a mplitude (square of angular fluctuation) in degrees.

square of the angular fluctuation; its validity is indicated by the fit of the straight lines to the data shown in Fig. 1.

For such a distribution, Yule and Kendall (1937) have shown that the mean value $|\overrightarrow{\Delta \theta}|$ of the magnitude of the vane fluctuations is related to the standard deviation $\sigma_{\Delta \theta}$ of the fluctuations, by

$$
|\overline{\Delta \theta}|=\frac{4}{5} \sigma_{\Delta \theta} .
$$

But in the notation of Harris and McCormick,

$$
|\overline{\Delta \theta}|=2 \theta / N,
$$

so that (1), (2), and (3) may be combined to give

$$
\sigma_{A}=\frac{12}{25} \sigma_{\Delta \theta} \cong \frac{1}{2} \sigma_{\Delta \theta} .
$$

Comparison of estimates based on the HarrisMcCormick technique with the alternative suggested here is shown in Fig. 2 for two series of runs.

The former method gives estimates as much as $50 \%$ higher than that based on the Gaussian distribution, chiefly as a result of the missing small amplitude oscillations. Similar bias must exist with data from the instrument designed by Humphrey and Wong ${ }^{2}$ (for

2 Humphrey, P. A., and L. J. Wong, 1961: An economical wind recording system. Paper presented at 191st meeting of the American Meteorological Society, 21-23 March, Chicago, Ill.

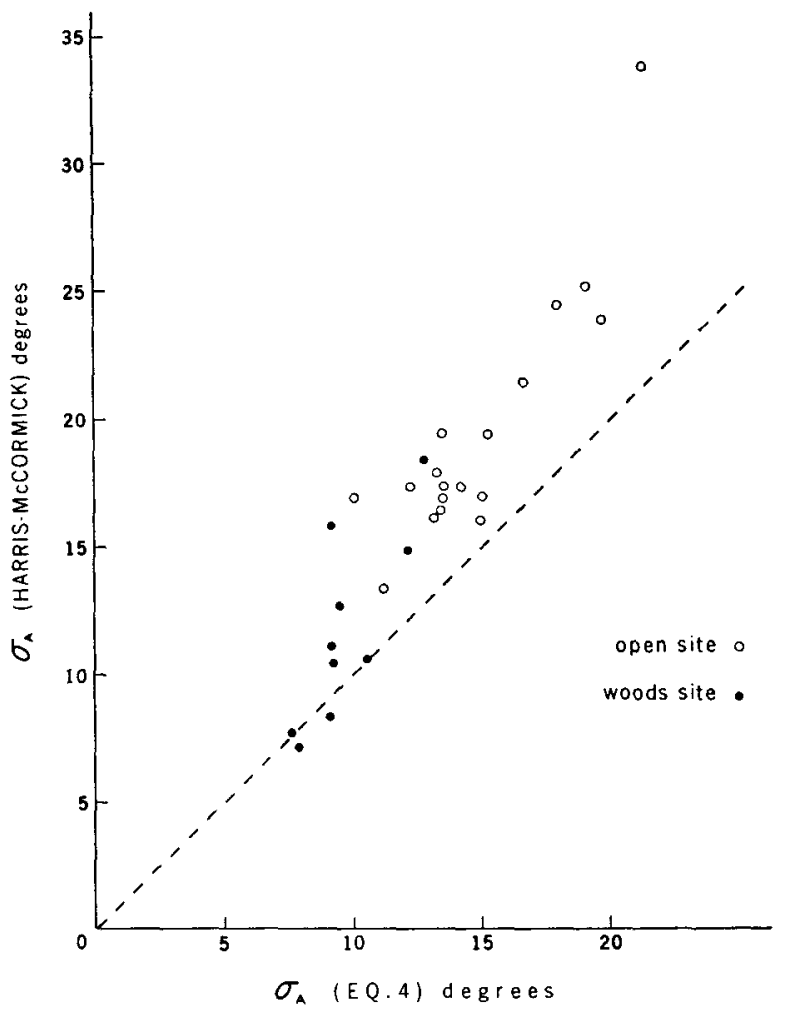

FIg. 2. Comparisons of estimates of the standard deviation $\sigma_{A}$ of the horizontal wind direction obtained from the Harris and McCormick (1963) technique [Eq. (1)] with that derived in this paper $[\mathrm{Eq} .(4)]$.

which the Harris-McCormick technique was developed), since small fluctuations are not counted.

The technique was developed as part of a flexible field study involving a minimum of equipment. It could also be used to advantage in calculating the standard deviation of azimuth angle from moderately high speed chart records of wind direction when these are available.

When the sampling is confined to alternating extreme values occurring at regular intervals, the ratio of the standard deviations in Eq. (4) can be shown to be $1 /[2(1-r)]^{\frac{1}{2}}$, where $r$ is the correlation coefficient between successive extreme values. For the vane oscillations considered here $r$ approaches the value -1 , so that the above ratio approaches $\frac{1}{2}$ as a limit, justifying the approximate value given by Eq. (4).

\section{REFERENCES}

Harris, E. K., and R. A. McCormick, 1963: A simple procedure for estimating the standard deviation of wind fluctuations. J. A ppl. Meteor., 2, 804-805.

Hay, J. S., and F. Pasquill, 1959: Diffusion from a continuous source in relation to the spectrum and scale of turbulence. Advances in Geopliysics, Vol. 6, New York and London, Academic Press, pp. 345-365.

MacCready, P. B., Jr., and H. R. Jex, 1964: Turbulent energy measurement by vanes. Quart. J. Roy. Meteor. Soc., 90, 198-203.

Singer, I. A., and M. E. Smith, 1953: Relationship of gustiness to other meteorological parameters. $J$. Meleor., 10, 121-126.

Yule, G. U., and M. G. Kendall, 1937: An Introduction to the Theory of Statistics. London, Chas Griffin and Co., p. 182. 